\title{
Diet and Nutrition of Healthcare Workers in COVID-19 Epidemic-Hubei, China, 2019
}

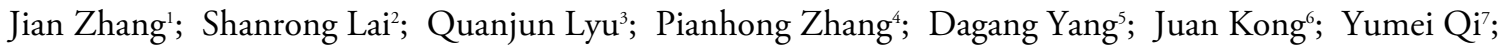 \\ Wei Yuan'; Shan Zeng;; Pengkun Song'; Titi Yang'; Li Li'; Jinglei Wang ${ }^{10}$; Yujing Liu ${ }^{10}$; \\ Tanxi Ge ${ }^{10}$; Qi Zhang ${ }^{10}$; Ganyu Feng'; Ailing Liu'; Gangqiang Ding, ${ }^{1, *}$
}

From late January to March, more than 42,000 doctors, nurses, public health workers, and health administrators across China went to Hubei Province to assist in the response to coronavirus disease 2019 (COVID-19). There are many studies on nutrition status of affected people amid natural disasters or pandemics (1-3), but the diet and nutrition status of healthcare workers receive little attention. The aim of this article is to provide evidence for addressing this issue by investigating food supply and nutrition status during the COVID-19 response period. The subjective survey was conducted from March 19 to April 1, 2020. The questionnaire composed of 9 questions including participants' occupation, body weight changes during the COVID-19 response, assessments of the amount and diversity of foods, meal tastes, changes in fitness due to consumption, dietary suggestions, and overall satisfaction for the meals. Healthcare workers randomly sampled from 27 provincial-level administrative divisions (PLADs) participated in the online survey excluding workers from Hubei Province, workers participating for less than 30 days in the COVID-19 response, and workers participating in the response in their hometown. A total of 1,048 valid responses were used in the final analysis. All statistical analyses were performed using SPSS 22.0 (SPSS, Inc.,
Chicago, IL, USA).

The participants were $57.9 \%$ females and $42.1 \%$ males, $30.7 \%$ doctors, $54.1 \%$ nurses, $11.2 \%$ public health workers, and $4.0 \%$ others. The age range was $22-60$ years, and $96.6 \%$ of the surveyed subjects were aged below 50 years. The average age was $37.6 \pm 7.3$ years for men and 33.8 \pm .2 years for women.

Participants with self-reported weight gain were $26.2 \%$ of the total including $27.4 \%$ of males and $25.4 \%$ of females, $27.6 \%$ of doctors, $25.2 \%$ of nurses, $26.5 \%$ of public health workers, and $28.6 \%$ of others. Participants with self-reported weight loss were $22.9 \%$ of the total including $20.0 \%$ of males and $25.0 \%$ of females, $19.3 \%$ of doctors, $26.1 \%$ of nurses, $17.9 \%$ of public health workers, and $21.4 . \%$ of others (Figure 1).

Nearly $65 \%$ of the healthcare workers thought that the box meals they were provided could meet their nutrition requirements, and more than $80 \%$ of them gave scores of $\geq 8$ point in a 10-point overall assessment of the meals (Figure 2) despite some complaints about the taste. Approximately $30 \%$ of the healthcare workers thought that the dishes were too greasy, and $16.8 \%$ of them complained that the dishes were too salty. The proportion suggesting reducing edible oil, salt, and red meat were $48.4 \%, 42.0 \%$, and $33.4 \%$, respectively. More than $50 \%$ of the healthcare workers

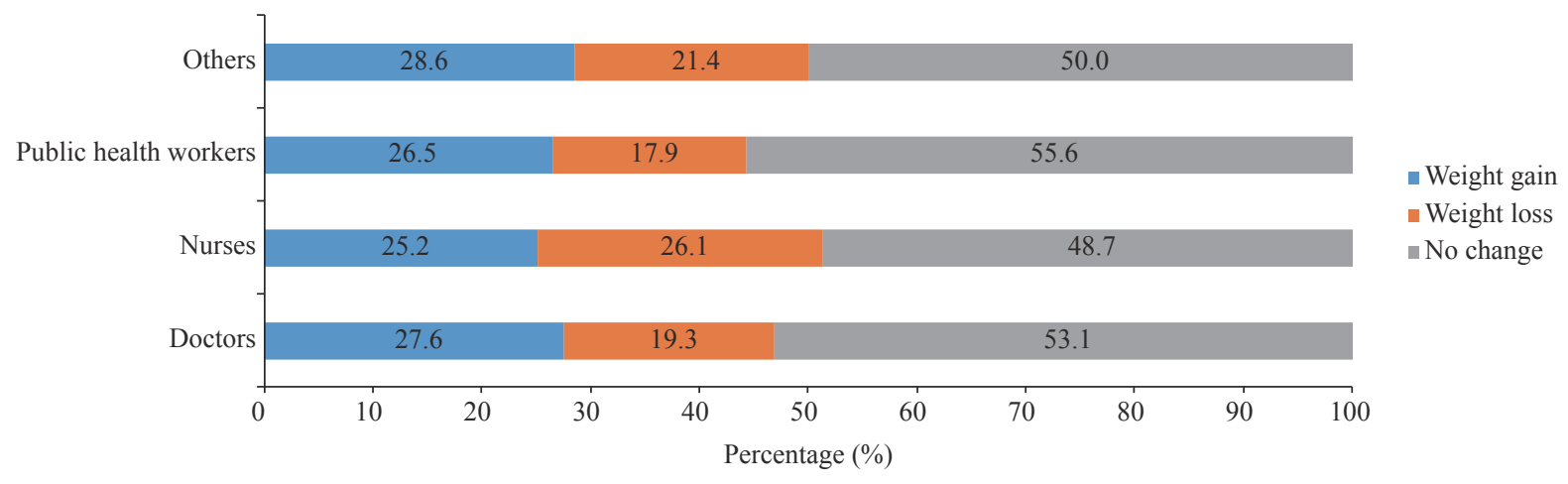

FIGURE 1. The percentage of self-reported body weight gain/loss/no change in healthcare workers during COVID-19 response in Hubei Province (\%). 


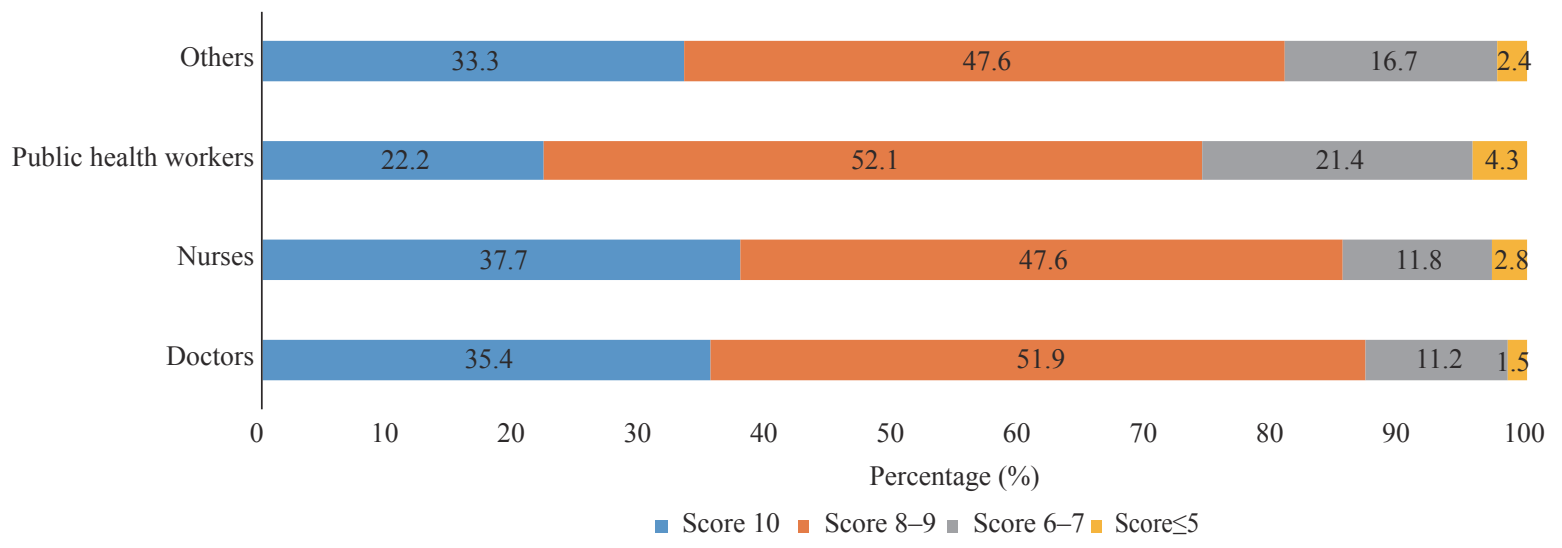

FIGURE 2. The percentage of assessment score from healthcare workers of overall satisfaction for meals during the COVID-19 response in Hubei Province.

asked for more vegetables, coarse grains, fruits, nuts, and soybeans.

As far as we know, this is the first survey on the diet and nutrition for healthcare workers during the pandemic. Our data showed that even in a short period of 1-2 months, participant body weight changed, which was an important nutritional index. Although self-reported, the results suggested that a large proportion of healthcare workers had unbalanced diets throughout the response. In addition, $13.5 \%$ of the healthcare workers complained of mild discomfort after consumption, and 8 persons, including 6 females and 2 males, experienced severe discomfort and needed medication.

Daily diet has great influence on the health of medical workers, especially during the response period with heavy work intensity and physical and mental exhaustion. Our results suggested that some dietary problems existed such as energy intake imbalance and excessive use of oil and salt (4) in the meals provided to healthcare workers. Therefore, more effective approaches should be taken to improve nutritional quality for workers in public health crises in the future.

Acknowledgments: Thanks to the participation of all COVID-19 response healthcare workers and strong support from the National Health Commission of the People's Republic of China. Thanks to all members of the epidemiological investigation team of China CDC for their efforts in this investigation. doi: $10.46234 / \mathrm{ccdcw} 2020.121$

\#Corresponding author: Gangqiang Ding, dinggq@chinacdc.cn.

\begin{abstract}
${ }^{1}$ National Institute for Nutrition and Health, Chinese Center for Disease Control and Prevention, Beijing, China; ${ }^{2}$ Fujian Disease Control and Prevention, Fuzhou, Fujian, China; ${ }^{3}$ Public Health College, Zhengzhou University, Zhengzhou, Henan, China; ${ }^{4}$ The Second Affiliated Hospital of Zhejiang University School of Medicine, Hangzhou, Zhejiang, China; ${ }^{5}$ Hospital affiliated to Guizhou Medical University, Guiyang, Guizhou, China; ${ }^{6}$ Shengjing Hospital of China Medical University, Shenyang, Liaoning, China; 7 Tianjin Third Central Hospital, Tian Jin, China; ${ }^{8}$ Zhabei Central Hospital of Jingan District, Shanghai, China; ${ }^{9}$ Jiang Su Province Hospital, Nanjing, Jiangsu, China; ${ }^{10}$ Chinese Center for Disease Control and Prevention, Beijing, China
\end{abstract}

Submitted: May 12, 2020; Accepted: June 11, 2020

\section{REFERENCES}

1. Aguayo VM, Sharma A, Subedi GR. Delivering essential nutrition services for children after the Nepal earthquake. Lancet Glob Health 2015;3(11): e665-6. https://www.ncbi.nlm.nih.gov/pubmed/?term= Aguayo+VM\%2C+Sharma+A\%2C+Subedi+GR.

2. Clark WA, Cress EM. Nutritional issues and positive living in human immunodeficiency virus/AIDS. Nurs Clin North Am 2018;53(1): 13-24. https://www.ncbi.nlm.nih.gov/pubmed/?term=William+Andrew+ Clark $\% 2 \mathrm{C} \% \mathrm{C} 2 \% \mathrm{~A} 0$ Eileen+M+Cress.

3. Zick SM, Snyder D, Abrams DI. Pros and cons of dietary strategies popular among cancer patients. Oncology 2018;32(11): 542-7. https:/www.ncbi.nlm.nih.gov/pubmed/?term=Zick+SM\%2C+Snyder+D $\% 2 \mathrm{C}+$ Abrams+DI.

4. Yu DM, He YN, Fang HY, Xu XL, Wang X, Yu WT, et al. Salt intake among Chinese adults in 2010-2012. Chin J Prev Med 2016;50(3): 217-20. https://www.ncbi.nlm.nih.gov/pubmed/26957237. (In Chinese). 